\title{
PI3 kinase directly phosphorylates Akt1/2 at Ser473/474 in the insulin signal transduction pathway
}

\section{A Tsuchiya, T Kanno and T Nishizaki}

Division of Bioinformation, Department of Physiology, Hyogo College of Medicine, 1-1 Mukogawa-cho, Nishinomiya 663-8501, Japan
Correspondence should be addressed to T Nishizaki

Email tomoyuki@hyo-med.ac.jp

\begin{abstract}
Insulin stimulated translocation of the glucose transporter GLUT4 from the cytosol to the plasma membrane in a concentration (1 nM-1 $\mu \mathrm{M}$ )-dependent manner and increased glucose uptake in 3T3-L1 adipocytes. Insulin-induced GLUT4 translocation to the cell surface was prevented by the phosphoinositide 3 kinase (PI3K) inhibitor wortmannin, the 3-phosphoinositide-dependent protein kinase 1 (PDK1) inhibitor BX912 or the Akt1/2 inhibitor MK2206, and by knocking-down PI3K, PDK1 or Akt1/2. Insulin increased phosphorylation of Akt1/2 at Thr308/309 and Ser473/474, to activate Akt1/2, in the adipocytes. Insulin-induced phosphorylation of Akt1/2 was suppressed by wortmannin and knockingdown PI3K, while no significant inhibition of the phosphorylation was obtained with BX912 or knocking-down PDK1. In the cell-free Akt assay, PI3K phosphorylated Akt1 both at Thr308 and Ser473 and Akt2 at Ser474 alone. In contrast, PDK1 phosphorylates Akt1 at Thr308 and Akt2 at Thr309. The results of this study indicate that PI3K activates Akt1, independently of PDK1, and Akt2 by cooperating with PDK 1 in the insulin signal transduction pathway linked to GLUT4 translocation.
\end{abstract}
Key Words
- adipocyte
- cell biology
- GLUT4
- insulin signaling

Journal of Endocrinology (2014) 220, 49-59

\section{Introduction}

Akt is a serine/threonine protein kinase bearing multiple cellular processes such as glucose metabolism, apoptosis, cell proliferation, transcription, and cell migration. Akt includes three closely related isoforms Akt1, Akt2, and Akt3 (Calera et al. 1998). Of the isoforms Akt1 exerts its anti-apoptotic action, thereby promoting cell survival (Song et al. 2005). Akt1 is also implicated in protein synthesis, responsible for skeletal muscle hypertrophy and tissue growth (Lai et al. 2004). Accumulating evidence has indicated the involvement of Akt1 in many types of cancer (Cheung \& Testa 2013). Akt2, on the other hand, plays a pivotal role in glucose homeostasis. Akt2 stimulates translocation of the glucose transporter GLUT4, abundantly expressed in skeletal muscle and fat cells, to the cell surface, causing insulin-induced glucose uptake into cells (Garofalo et al. 2003). Akt3 is preferentially expressed in the brain, but its role is not fully understood.

The receptor tyrosine kinase insulin receptor is implicated in the activation of Akt through a pathway along an insulin receptor substrate (IRS)/phosphatidylinositol 3 kinase (PI3K)/3-phosphoinositide-dependent protein kinase 1 (PDK1)/Akt axis (Maarbjerg et al. 2011). Insulin activates insulin receptor, to phosphorylate its own receptor and IRS, thereby dissociating IRS from insulin receptor to activate PI3K. The activated PI3K produces phosphatidylinositol (3,4,5)-triphosphate 
(PI $\left.(3,4,5) \mathrm{P}_{3}\right)$ by phosphorylation of phosphatidylinositol 4,5-bisphosphate $\left(\mathrm{PI}(4,5) \mathrm{P}_{2}\right)$, and in turn, $\mathrm{PI}(3,4,5) \mathrm{P}_{3}$ activates PDK1 through its binding. The activation of Akt $1 / 2$ is achieved by phosphorylation of Thr308 for Akt 1 and Thr309 for Akt2 in the activation-loop of the kinase domain and Ser473 for Akt1 and Ser474 for Akt2 in the carboxy-terminal regulatory region (Yang et al. 2002, Song et al. 2005). Thr308/309 is phosphorylated by PDK1, which is dephosphorylated by protein phosphatase $2 \mathrm{~A}$ (PP2A; Vanhaesebroeck \& Alessi 2000, Beaulieu et al. 2005). The phosphatase and tensin homolog (PTEN) and Src homology 2-containing inositol phosphatase (SHIP) reduce $\mathrm{PI}(3,4,5) \mathrm{P}_{3}$ by dephosphorylating it into $\mathrm{PI}(4,5) \mathrm{P}_{2}$ and $\operatorname{PI}(3,4) \mathrm{P}_{2}$, respectively, to attenuate PDK1 activity followed by Akt1/2 phosphorylation at Thr308/309 (Finlay 2012). The mammalian target of rapamycin complex 2 (mTORC2), that is activated by PI3K (Razmara et al. 2013), phosphorylates Akt1/2 at Ser473/474, and the $\mathrm{PH}$ domain and leucine-rich repeat protein phosphatase dephosphorylates Akt1/2 at this residue (Bayascas \& Alessi 2005, Gao et al. 2005). Phosphorylation of Akt $1 / 2$ at Ser473/474, alternatively, is achieved by an inhibitor of NFא-B kinase subunit $\varepsilon$ (IKBKE; Guo et al. 2011) or P21-activated kinase 1 (Pak1; Mao et al. 2008), in a PI3K-independent manner.

This study was conducted to gain further insight into PI3K-induced Akt activation in the insulin signal transduction pathway. To address this, we monitored GLUT4 mobilizations, measured glucose uptake into cells, and assayed Akt1/2 activity in 3T3-L1 adipocytes expressing myc-tagged GLUT4 (3T3-L1-GLUT4myc adipocytes) and under cell-free conditions. We show here that insulin stimulates GLUT4 translocation to the cell surface and increases glucose uptake into 3T3-L1 adipocytes in an Akt1/2-dependent manner; PI3K phosphorylates both Thr308 and Ser473 for Akt1 and Ser474 alone for Akt2; and PDK1 phosphorylates Thr308 for Akt1 and Akt2. This may represent fresh insight into PI3K-regulated Akt1/2 activation in the insulin signal transduction pathway.

\section{Subjects and methods}

\section{Cell culture}

3T3-L1-GLUT4myc fibroblast cell line expressing GLUT4myc is constructed by inserting a human c-MYC epitope (14 amino acids) into the first ectodomain of GLUT4. Cells were cultured in DMEM supplemented with $10 \%(\mathrm{v} / \mathrm{v})$ calf serum, penicillin (final concentration, $100 \mathrm{U} / \mathrm{ml}$ ), and streptomycin (final concentration, $0.1 \mathrm{mg} / \mathrm{ml}$ ), in a humidified atmosphere of $5 \% \mathrm{CO}_{2}$ and $95 \%$ air at $37^{\circ} \mathrm{C}$. When cells had reached confluence (day 0), medium was changed to DMEM supplemented with $10 \%(\mathrm{v} / \mathrm{v})$ fetal bovine serum (FBS), $1 \mu \mathrm{M}$ dexamethasone, $0.5 \mathrm{mM}$ 3-isobutyl-metyhl-xanthine, and $0.1 \mathrm{mg} / \mathrm{ml}$ insulin to differentiate from fibroblast to adipocytes (3T3-L1-GLUT4myc adipocytes). At day 3 , day 7 , and day 11 , the medium was changed to DMEM supplemented with $10 \%$ (v/v) FBS. At day 14, cells were used for experiments. 3T3-L1GLUT4myc adipocytes were used for all the cell experiments in this study.

\section{Oil-Red $\mathrm{O}$ staining of 3T3-L1 adipocytes}

Oil-Red O (Wako Pure Chemical Industries, Osaka, Japan) was dissolved in isopropanol and left over night at room temperature. The solution was diluted with distilled water (6:4) and filtered through a filter paper. Cells on day 0 , day 3 , and day 14 from differentiation treatment were fixed with $10 \%(\mathrm{v} / \mathrm{v})$ formalin in PBS for $10 \mathrm{~min}$ and stained with the Oil-Red O solution for $20 \mathrm{~min}$ at room temperature. Cells were washed with $60 \%(\mathrm{v} / \mathrm{v})$ isopropanol and then washed twice with PBS. Fat droplets in adipocytes were visualized with a phase contrast microscope.

\section{Monitoring of GLUT4 mobilizations}

3T3-L1-GLUT4myc adipocytes were incubated in KrebsRinger-HEPES buffer $(136 \mathrm{mM} \mathrm{NaCl}, 4.7 \mathrm{mM} \mathrm{KCl}$, $1.25 \mathrm{mM} \mathrm{CaCl}_{2}, 1.25 \mathrm{mM} \mathrm{MgSO}_{4}$, and $20 \mathrm{mM}$ HEPES, $\mathrm{pH}$ 7.5) containing $0.2 \%(\mathrm{w} / \mathrm{v})$ BSA supplemented with $10 \mathrm{mM}$ glucose for $1 \mathrm{~h}$ at $37^{\circ} \mathrm{C}$. Cells were treated with insulin in the presence and absence of inhibitors for $20 \mathrm{~min}$. Then, cells were homogenized by sonication in an ice-cold mitochondrial buffer $(210 \mathrm{mM}$ mannitol, $70 \mathrm{mM}$ sucrose, and $1 \mathrm{mM}$ EDTA, $10 \mathrm{mM}$ HEPES, pH 7.5) containing $1 \%(\mathrm{v} / \mathrm{v})$ protease inhibitor cocktail (Nacalai Tesque, Kyoto, Japan) and subsequently, homogenates were centrifuged at $800 \mathrm{~g}$ for $5 \mathrm{~min}$ at $4^{\circ} \mathrm{C}$. The supernatants were centrifuged at $11000 \mathrm{~g}$ for $15 \mathrm{~min}$ at $4{ }^{\circ} \mathrm{C}$ and further, the collected supernatants were ultracentrifuged at $100000 \boldsymbol{g}$ for $60 \mathrm{~min}$ at $4{ }^{\circ} \mathrm{C}$ to separate the cytosolic and plasma membrane fraction. The supernatants and pellets were used as the cytosolic and plasma membrane fractions respectively. Whether the cytosolic and plasma membrane components were successfully separated was confirmed in the western blot analysis using antibodies against the cytosolic marker, lactate dehydrogenase, and the plasma membrane marker, cadherin.

Published by Bioscientifica Ltd 
Protein concentrations for each fraction were determined using a BCA protein assay kit (Thermo Fisher Scientific, Waltham, MA, USA). Plasma membrane fraction proteins were resuspended in the mitochondrial buffer containing $1 \%(\mathrm{w} / \mathrm{v})$ SDS. Proteins for each fraction were separated by SDS-PAGE and transferred to polyvinylidene difluoride (PVDF) membranes. After blocking with TBS-T (150 mM NaCl, 0.1\% (v/v) Tween-20, and $20 \mathrm{mM}$ Tris, $\mathrm{pH}$ 7.5) containing 5\% (w/v) BSA, blotting membranes were reacted with an anti-c-myc antibody (Merck Millipore) followed by an HRP-conjugated goat anti-mouse IgG antibody. Immunoreactivity was detected with an ECL kit (Invitrogen) and visualized using a chemiluminescence detection system (GE Healthcare, Piscataway, NJ, USA). Signal density was measured with ImageQuant software (GE Healthcare).

\section{Glucose uptake assay}

3T3-L1-GLUT4myc adipocytes were incubated in the Krebs-Ringer-HEPES buffer containing $0.2 \%(\mathrm{w} / \mathrm{v})$ BSA supplemented with $10 \mathrm{mM}$ glucose for $1 \mathrm{~h}$ at $37^{\circ} \mathrm{C}$. Then, cells were left untreated or treated with insulin $(100 \mathrm{nM})$ in PBS supplemented with $10 \mathrm{mM}$ glucose for $20-120 \mathrm{~min}$ at $37^{\circ} \mathrm{C}$. After treatment, extracellular solution was collected and glucose was labeled with $p$-aminobenzoic ethyl ester (ABEE). Then, $5 \mu \mathrm{l}$ ABEE-labeled solution was injected onto the column $(150 \times 4.6 \mathrm{~mm})$ equipped in the HPLC system. ABEE-labeled glucose was detected at an excitation wavelength of $305 \mathrm{~nm}$ and an emission wavelength of $360 \mathrm{~nm}$ using a fluorescence detector. The concentration of glucose taken up into cells was calculated by subtracting extracellular glucose concentration after incubation for 20-120 min from initial extracellular glucose concentration $(10 \mathrm{mM})$.

\section{Construction and transfection of siRNA}

The siRNA to silence the Akt1/2-targeted gene (Akt1/2 siRNA) was obtained from Santa Cruz Biotechnology, Inc. The siRNAs to silence the PI3K p85 $\alpha$-targeted gene (PI3K KD), the PDK1-targeted gene (PDK1 KD), and the negative control siRNA (NC) were obtained from Ambion (Carlsbad, CA, USA). The sequences of siRNAs used were $5^{\prime}$-GCGAAUGAUAUGUAUCAGAtt- $3^{\prime}$ and $5^{\prime}$-UCUGAUACAUAUCAUUCGCtc-3' for PI3K p85 $\alpha, 5^{\prime}$-CCUCGUUUAUGUUUCUGCGtt- $3^{\prime}$ and 5'-CGCAGAAACAUAAACGAGGtc-3' for PDK1. Each NC siRNA had the scrambled sequence, the same GC content, and nucleic acid composition. siRNAs were transfected into the cells using a Lipofectamine reagent (Invitrogen). Cells were used for experiments $48 \mathrm{~h}$ after transfection.

\section{Western blotting}

3T3-L1-GLUT4myc adipocytes were incubated in KrebsRinger-HEPES buffer containing $0.2 \%(\mathrm{w} / \mathrm{v})$ BSA, supplemented with $10 \mathrm{mM}$ glucose for $1 \mathrm{~h}$ at $37^{\circ} \mathrm{C}$. The cells transfected with and without siRNAs were left untreated or treated with insulin in the presence and absence of inhibitors for $10 \mathrm{~min}$. Next, the cells were lysed in lysis buffer $(150 \mathrm{mM} \mathrm{NaCl}, 20 \mathrm{mM}$ EDTA, $0.5 \%(\mathrm{v} / \mathrm{v})$ Nonidet P-40, and $50 \mathrm{mM}$ Tris, pH 7.4) containing $1 \%$ $(\mathrm{v} / \mathrm{v})$ protease inhibitor cocktail and $1 \%(\mathrm{v} / \mathrm{v})$ phosphatase inhibitor cocktail (Nacalai Tesque), and then centrifuged at $800 \mathrm{~g}$ for $5 \mathrm{~min}$ at $4{ }^{\circ} \mathrm{C}$. The supernatant was used as total cell lysate.

For western blotting, proteins were separated by SDS-PAGE and then transferred to PVDF membranes. Blotting membranes were blocked with TBS-T containing $5 \%(\mathrm{w} / \mathrm{v})$ BSA and subsequently reacted with antibodies against peroxisome proliferator-activated receptor $\gamma$ (PPAR $\gamma$ ) (Cell Signaling Technology, Inc., Danvers, MA, USA), phospho-Thr308/309-Akt1/2 (pT308(9)), phosphoSer473/474-Akt1/2 (pS473(4)), Akt1/2 (Cell Signaling Technology), Akt1 (Cell Signaling Technology), Akt2 (Cell Signaling Technology), PI3K (Sigma), PDK1 (Sigma), or $\beta$-actin (Sigma). After washing, membranes were reacted with an HRP-conjugated goat anti-rabbit IgG or goat anti-mouse IgG antibody. Immunoreactivity was detected with an ECL kit (Invitrogen) and visualized using a chemiluminescence detection system (GE Healthcare). Protein concentrations for each sample were determined with a BCA protein assay kit (Thermo Fisher Scientific).

\section{Cell-free Akt assay}

Human recombinant Akt1 or human recombinant Akt2 (Active Motif, Carlsbad, CA, USA) was reacted without or with His-tagged human recombinant PI3K (p110ß/p85 $\alpha$ ) (Sigma) or His-tagged human recombinant PDK1 (SignalChem, Richmond, BC, Canada), which was purified by affinity chromatography, in the presence and absence of wortmannin or BX912 in a medium containing $25 \mathrm{mM}$ 3-morpholinopropanesulfonic acid ( $\mathrm{pH}$ 7.2), $25 \mathrm{mM} \mathrm{MgCl}_{2}, 12.5 \mathrm{mM}$ glycerol 2-phosphate, $5 \mathrm{mM}$ ethylene glycol-bis(2-aminoethyl ether)- $N, N, N^{\prime}, N^{\prime}$ tetraacetic acid, $2 \mathrm{mM}$ EDTA, $0.25 \mathrm{mM}$ dithiothreitol, and $250 \mu \mathrm{M}$ ATP at $30{ }^{\circ} \mathrm{C}$ for $20 \mathrm{~min}$. Phosphorylated Akt was detected by western blotting using antibodies against pT308(9), pS473(4), and Akt1/2 as described earlier.

Published by Bioscientifica Ltd 


\section{Statistical analysis}

Statistical analysis was carried out using unpaired $t$-test, Dunnett's test, and ANOVA followed by a Bonferonni correction.

\section{Results}

Insulin stimulates GLUT4 translocation to the plasma membrane and increases glucose uptake into cells in an Akt1/2-dependent manner

We initially examined whether 3T3-L1-GLUT4myc fibroblasts are differentiated into adipocytes using Oil-Red O. Adipose tissue is stained with Oil-Red $\mathrm{O}$, to detect adipose conversion (Ramírez-Zacarías et al. 1992). 3T3-L1-GLUT4myc fibroblasts before differentiation induction (day 0), with spindle-like shapes, had no reaction to Oil-Red O (Fig. 1A). On day 3 after differentiation induction a few cells, which had assumed a round shape, were positive to Oil-Red $\mathrm{O}$ and on day 14 almost all the cells, which had assumed a round shape, were dyed red (Fig. 1A). This indicates that 3T3-L1-GLUT4myc fibroblasts are readily differentiated into adipocytes.

To obtain further evidence for adipocyte differentiation, we carried out western blotting using an antibody against PPAR $\gamma$, an adipocyte-specific marker (Tontonoz et al. 1994). Expression of PPAR $\gamma 1$ and PPAR $\gamma 2$ was gradually increased after differentiation induction, and the expression on day 14 after differentiation induction was significantly much higher than that on day 0 (Fig. 1B). This confirms that 3T3-L1-GLUT4myc fibroblasts are actually differentiated into adipocytes on day 14 after differentiation induction. We, therefore, used 3T3-L1-GLUT4myc fibroblasts differentiated for more than 14 days (3T3-L1GLUT4myc adipocytes) for the ensuing experiments.

Insulin promoted GLUT4 translocation from the cytosol to the plasma membrane in 3T3-L1-GLUT4myc adipocytes in a concentration $(1 \mathrm{nM}-1 \mu \mathrm{M})$-dependent manner (Fig. 2A). In the glucose assay, insulin (100 nM) significantly increased glucose uptake into 3T3-L1GLUT4myc adipocytes in a treatment time (20-120 min)-dependent manner, the extent reaching approximately twofold more than control levels at 120 min (Fig. 2B). Collectively, these results indicate that insulin stimulates GLUT4 translocation to the cell surface and promotes glucose uptake into adipocytes.

Akt plays a central role in insulin-stimulated GLUT4 translocation to the cell surface. The amount of expression of Akt1 and Akt2 in differentiated 3T3-L1-GLUT4myc adipocytes was calculated from the Akt1 and Akt2

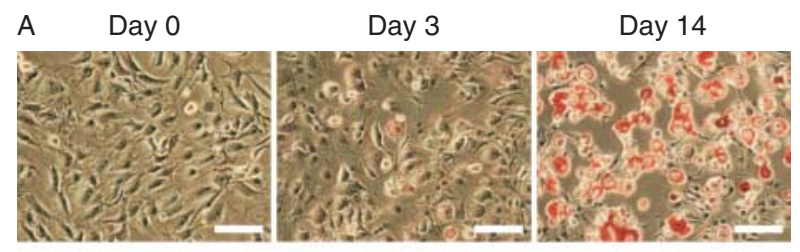

B
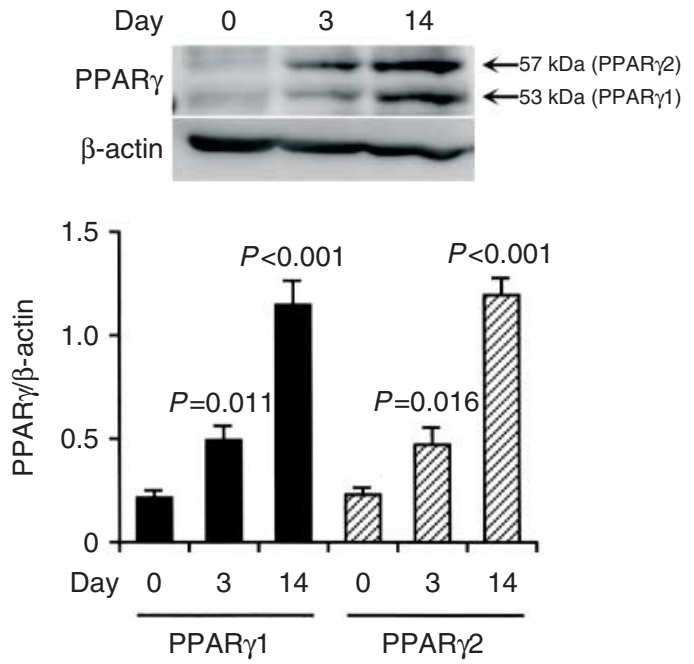

Figure 1

(A) Oil-Red O staining. 3T3-L1-GLUT4myc fibroblasts were stained with Oil-Red $\mathrm{O}$ on day 0 , day 3 , and day 14 after differentiation induction. Bars, $100 \mu \mathrm{m}$. Note that adipose droplets in cells are dyed red and that a similar effect was found in four independent experiments. (B) Cells collected on day 0 , day 3 , and day 14 after differentiation induction were lysed followed by western blotting using antibodies against PPAR $\gamma$ and $\beta$-actin. In the blotting membrane shown, an anti-PPAR $\gamma$ antibody produces two signal bands at 53 and $57 \mathrm{kDa}$, each corresponding to PPAR $\gamma 1$ and PPAR $\gamma 2$. Signal intensities for PPAR $\gamma 1$ and PPAR $\gamma 2$ were normalized to those for $\beta$-actin. In the graph, each column represents the mean ( \pm S.E.M.) normalized expression of PPAR $\gamma 1$ and PPAR $\gamma 2$ ( $n=4$ independent experiments). $P$ values as compared with the expression on day 0, Dunnett's test.

concentration/intensity standard curve, respectively. As previously shown (Calera et al. 1998), 3T3-L1-GLUT4myc adipocytes expressed both Akt1 and Akt2, but the expression level of Akt2 $(18.016 \pm 1.487 \mathrm{ng} / \mu \mathrm{l})$ was much greater than that of Akt1 $(0.083 \pm 0.005 \mathrm{ng} / \mu \mathrm{l})$ (Fig. 3A).

The insulin (100 nM)-induced increase in the GLUT4 localization on the cell surface was abolished by the Akt1/2 inhibitor MK2206 (5 $\mu \mathrm{M})$ (Fig. 3B), indicating that insulin promotes GLUT4 translocation to the cell surface in an Akt1/2-dependent manner. To obtain further evidence for this, we constructed the Akt $1 / 2$ siRNA. In the western blot analysis, expression of Akt1/2 protein was apparently reduced in 3T3-L1-GLUT4myc adipocytes transfected with the Akt1/2 siRNA compared with that for cells transfected with the NC siRNA (Fig. 3C), confirming Akt1/2 knock-down. The insulin (100 nM)-induced

Published by Bioscientifica Ltd 
A
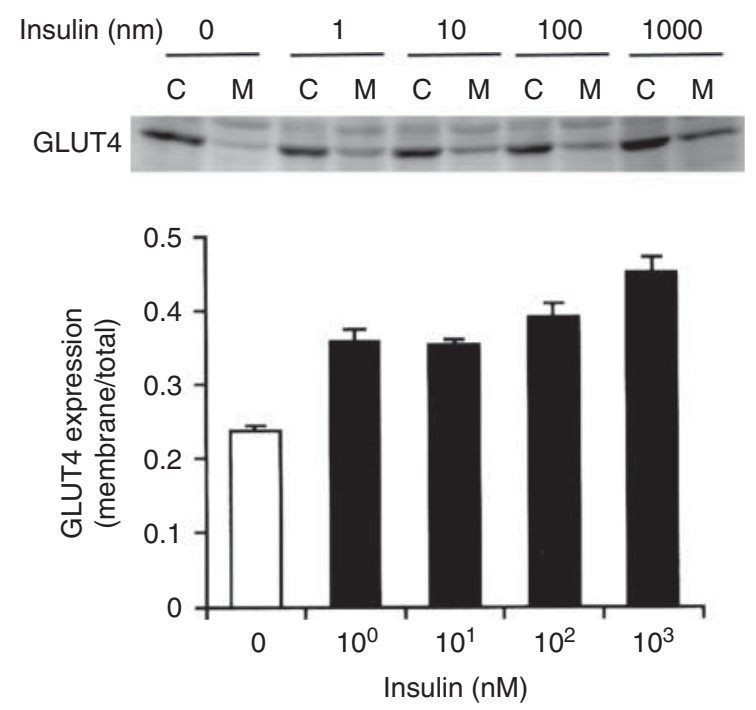

B

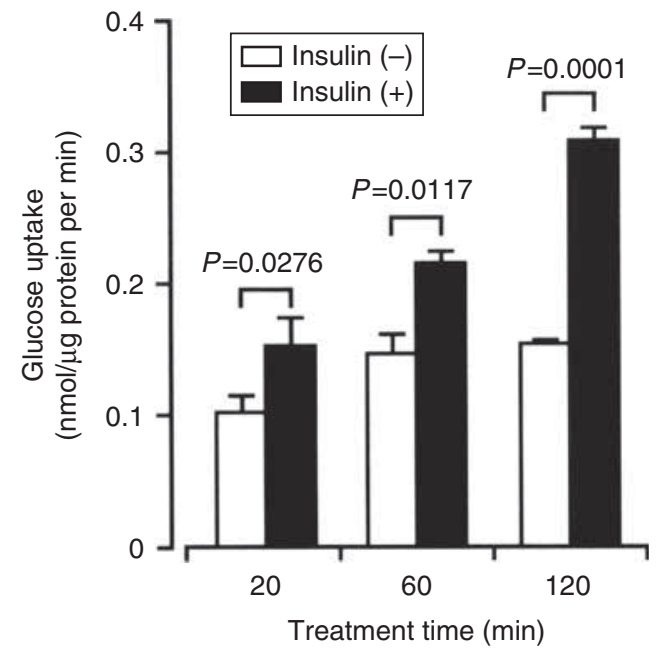

Figure 2

Insulin stimulates GLUT4 translocation toward the cell surface and increases glucose uptake into 3T3-L1-GLUT4myc adipocytes. (A) Cells were left untreated or treated with insulin at the concentrations indicated for $20 \mathrm{~min}$. Then, cells were lysed and separated into the cytosolic (C) and plasma membrane fractions (M), followed by western blotting using antibody against c-myc. In the graph, each column represents the mean ( \pm S.E.M.) ratio of signal intensity for c-myc in the plasma membrane fraction to that in the total cell ( $n=4$ independent experiments). (B) Cells were incubated in PBS containing glucose $(10 \mathrm{mM})$ for periods of time as indicated in the presence and absence of insulin (100 nM), and then extracellular glucose was measured by HPLC. In the graph, each column represents the mean ( \pm s.E.M.) glucose uptake (nmol/ $\mu \mathrm{g}$ protein per $\mathrm{min}$ ) ( $n=4$ independent experiments). $P$ values, unpaired $t$-test.

increase in the GLUT4 localization on the cell surface was significantly suppressed by knocking-down Akt1/2 (Fig. 3C). Taken together, these results indicate that Akt1/2 is a key factor for insulin-stimulated GLUT4 translocation to the cell surface.
PI3K phosphorylates both Thr308 and Ser473 for Akt1 and Ser474 alone for Akt2

Insulin $(100 \mathrm{nM})$ increased phosphorylation of Akt1/2 both at Thr308/309 and Ser473/474, indicating that insulin activates Akt1/2 in 3T3-L1-GLUT4myc adipocytes (Fig. 4A and B). Insulin-induced phosphorylation of Akt1/2 at Thr308/309 and Ser473/474 was clearly inhibited by the PI3K inhibitor wortmannin (20 nM) (Fig. 4A). In contrast, the PDK1 inhibitor BX912 (100 nM) also reduced insulininduced phosphorylation of Akt1/2 at Thr308/309 and Ser473/474, but not significantly (Fig. 4B). These results indicate that in response to insulin PI3K could still activate Akt1/2 in the absence of PDK1 in adipocytes.

To knock-down PI3K and PDK1, we constructed siRNAs for PI3K and PDK1. In the western blot analysis, expression of PI3K and PDK1 proteins was significantly reduced in 3T3-L1-GLUT4myc adipocytes transfected with the PI3K siRNA and the PDK1 siRNA, respectively, as compared with that for cells transfected with each NC siRNA (Fig. 5A and B), confirming knocking-down of PI3K and PDK1. Insulin (100 nM)-induced phosphorylation of Akt1/2 at Thr308/309 and Ser473/474 was apparently suppressed by knocking-down PI3K (Fig. 5A), but otherwise no inhibition was obtained by knocking-down PDK1 (Fig. 5B). This further supports the notion that insulin stimulates Akt $1 / 2$ activation in a PI3K-dependent manner, without PDK1, in adipocytes.

In the cell-free assay, PI3K $(1 \mu \mathrm{g} / \mathrm{ml})$ phosphorylated Akt1 both at Thr308 and Ser473, which was abrogated by wortmannin $(20 \mathrm{nM})$ (Fig. 6A). In contrast, PDK1 $(1 \mu \mathrm{g} / \mathrm{ml})$ phosphorylated Akt1 only at Thr308, which was abolished by BX912 (100 nM) (Fig. 6B). Collectively, these results indicate that PI3K is capable of activating Akt1 still in the absence of PDK1. PI3K $(1 \mu \mathrm{g} / \mathrm{ml})$, on the other hand, phosphorylated Akt2 at Ser474 alone, and the effect was abolished by wortmannin (20 nM) (Fig. 6C). PDK1 $(1 \mu \mathrm{g} / \mathrm{ml})$ phosphorylated Akt2 at Thr309 alone, and the effect was completely inhibited by BX912 (100 nM) (Fig. 6D). The activation of Akt2, accordingly, appears to be achieved by PI3K and PDK1.

\section{PDK1 as well as PI3K also participate in the regulation of GLUT4 translocation to the cell surface}

Finally, we examined whether PI3K and PDK1 regulate GLUT4 translocation to the cell surface. Insulin (100 nM)stimulated GLUT4 translocation to the cell surface in 3T3L1-GLUT4myc adipocytes was significantly inhibited by wortmannin (20 nM) (Fig. 7A) or knocking-down PI3K

Published by Bioscientifica Ltd 
A
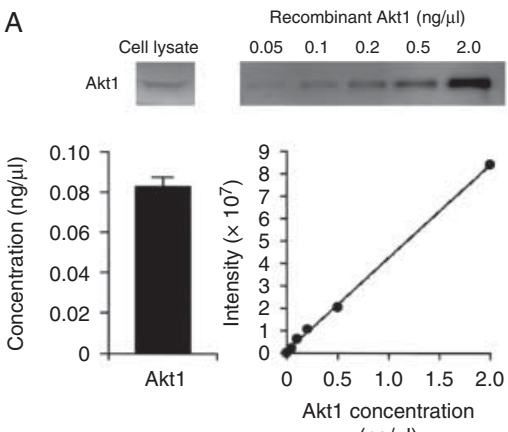

(ng/ul)
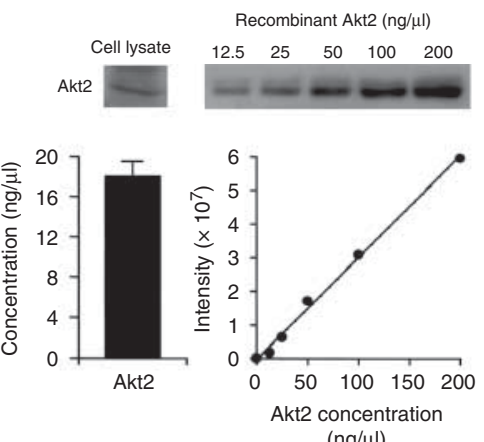

$(\mathrm{ng} / \mu \mathrm{l})$

B

$$
M \frac{+M K}{C M} C M \frac{\text { Insulin }}{C M}
$$

C

\section{Figure 3}

Insulin stimulates GLUT4 translocation toward the plasma membrane in an Akt1/2-dependent manner. (A) The Akt1 or Akt2 concentration/intensity standard curves were made using a human recombinant Akt1 or Akt2 respectively. 3T3-L1-GLUT4myc fibroblasts on day 14 after differentiation induction were lysed followed by western blotting using antibodies against Akt1 and Akt2, and the amount of Akt1 and Akt2 was calculated from each standard curve ( $n=4$ independent experiments). (B) 3T3-L1GLUT4myc adipocytes were treated with insulin $(100 \mathrm{nM})$ in the presence and absence of MK2206 (+MK) $(5 \mu \mathrm{M})$. Then, cells were lysed and separated into the cytosolic (C) and plasma membrane fractions (M), followed by western blotting using an antibody against c-myc. In the graph, each column represents the mean ( \pm S.E.M.) ratio of signal intensity for c-myc in the plasma membrane fraction to that in the total cell $(n=4$ independent experiments). $P$ values, ANOVA followed by a Bonferonni correction. NS, not significant. (C) In the left panel, 3T3-L1-GLUT4myc adipocytes were transfected with the NC siRNA or the Akt1/2 siRNA, and $48 \mathrm{~h}$ after transfection western blotting was carried out using antibodies against Akt $1 / 2$ or $\beta$-actin. Signal intensities for Akt1/2 were normalized to those for $\beta$-actin. In the graph, each column represents the mean ( \pm s.E.M.) normalized expression of Akt1/2 ( $n=4$ independent experiments). $P$ value, unpaired $t$-test. In the right panel, cells transfected with the NC siRNA (NC) or the Akt1/2 siRNA (Akt1/2 KD) were left untreated or treated with insulin (100 $\mathrm{nM})$ for $20 \mathrm{~min}$. Then, cells were lysed and separated into the cytosolic (C) and plasma membrane fractions (M), followed by western blotting using an antibody against c-myc. In the graph, each column represents the mean ( \pm s.E.M.) ratio of signal intensity for c-myc in the plasma membrane fraction to that in the total cell ( $n=4$ independent experiments). $P$ values, ANOVA followed by a Bonferonni correction. NS, not significant.
(Fig. 7C). Moreover, the insulin (100 nM)-induced increase in the GLUT4 localization on the cell surface was also prevented by BX912 (100 nM) (Fig. 7B) or knocking-down PDK1 (Fig. 7D). Taken together, these results indicate that PI3K and PDK1, a downstream effector of PI3K, are engaged in the insulin-stimulated GLUT4 translocation to the cell surface.

\section{Discussion}

GLUT4 is a member of the facilitative glucose transporter family, characterized by preferential expression in fat and muscle tissues, where it is responsible for insulininduced glucose uptake into cells. GLUT4 is unique among other members of the GLUT family in its dynamic cycling within adipocytes and muscle cells. Insulin stimulates GLUT4 translocation to the cell surface by activating insulin receptor, and when insulin receptor is inactivated, GLUT4 is rapidly removed from the plasma membrane into the cytosol and accumulated in intracellular organelles such as the trans-Golgi network, recycling endosome, and diverse tubulo-vesicular bodies (Bryant et al. 2002, Rudich \& Klip 2003, Watson et al. 2004, Ishiki \& Klip 2005).

$$
\begin{aligned}
& \text { http://joe.endocrinology-journals.org } \\
& \text { DOI: } 10.1530 / J O E-13-0172
\end{aligned}
$$

(๑) 2014 The authors Printed in Great Britain 
A
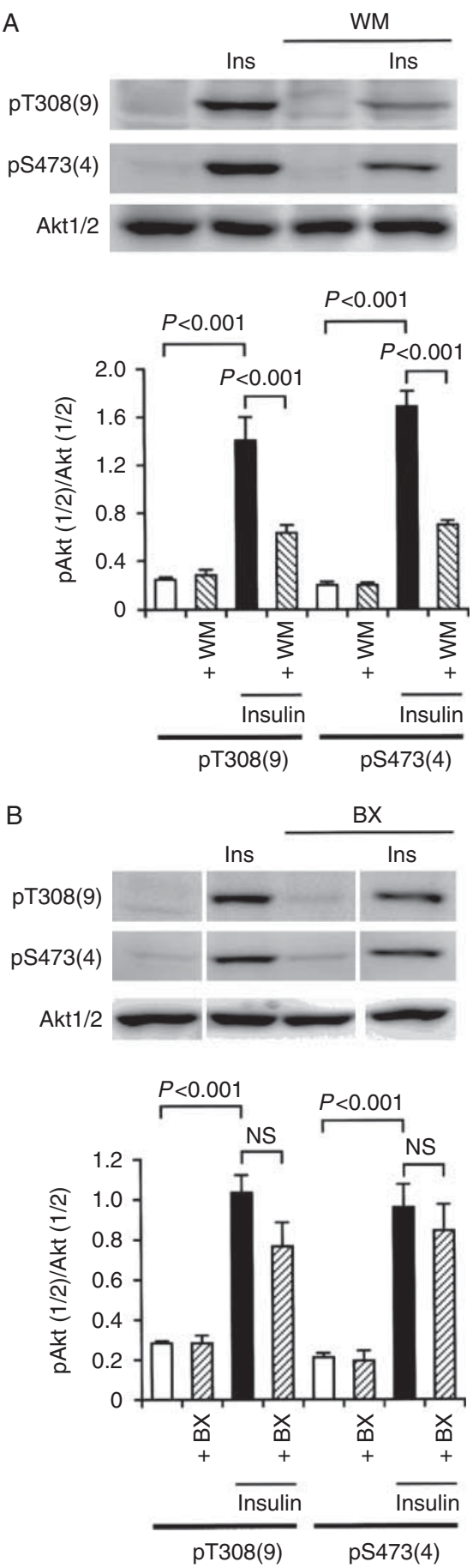

\section{Figure 4}

Insulin stimulates phosphorylation of Akt1/2 in a PI3K-dependent manner in 3T3-L1-GLUT4myc adipocytes. Cells were left untreated or treated with insulin (Ins) $(100 \mathrm{nM})$ for $10 \mathrm{~min}$ in the presence and absence of wortmannin (+WM) $(20 \mathrm{nM})(\mathrm{A})$ or BX912 (+BX) $(100 \mathrm{nM})(\mathrm{B})$. Western blotting was carried out using antibodies against pT308(9), pS473(4), and Akt1/2. Signal intensities for phosphorylated Akt1/2 (pAkt1/2) were normalized to those for Akt1/2. In the graphs, each column represents the mean ( \pm s.E.M.) normalized intensity for pAkt $1 / 2$ at each site $(n=4$ independent experiments). $P$ values, ANOVA followed by a Bonferonni correction. NS, not significant.
Akt is a key regulator for insulin-regulated GLUT4 translocation to the cell surface (Cong et al. 1997, Wang et al. 1999). In this study, insulin promoted GLUT4 translocation from the cytosol to the plasma membrane in a concentration $(1 \mathrm{nM}-1 \mu \mathrm{M})$-dependent manner and increased glucose uptake into 3T3-L1-GLUT4myc adipocytes. Insulin-induced GLUT4 translocation to the cell surface was clearly inhibited by the Akt $1 / 2$ inhibitor MK2206 or knocking-down Akt1/2, indicating that Akt1/2 is required for insulin-stimulated GLUT4 translocation to the cell surface. Accumulating evidence has indicated that Akt is activated via a pathway along an insulin receptor/IRS/PI3K/PDK1/Akt axis (Ishikura et al. 2008). Akt $1 / 2$ is activated through its phosphorylation at Thr308/309 in the activation-loop of the kinase domain and Ser473/474 in the carboxy-terminal regulatory region (Yang et al. 2002, Song et al. 2005). In this study, insulin increased phosphorylation of Akt1/2 both at Thr308/309 and Ser473/474 in 3T3-L1-GLUT4myc adipocytes, confirming that insulin activates Akt1/2. Surprisingly, insulin-induced phosphorylation of Akt1/2 was abrogated by the PI3K inhibitor wortmannin or knocking-down PI3K in 3T3-L1-GLUT4myc adipocytes, yet it was not significantly inhibited by the PDK1 inhibitor BX912 or knockingdown PDK1. This suggests that PI3K is indispensable for insulin-induced activation of Akt1/2. In the cell-free assay, PI3K phosphorylated Akt1 both at Thr308 and Ser473, but PDK1 phosphorylated Akt1 only at Thr308. This provides direct evidence that PI3K has the potential to activate Akt1 in a PDK1-independent manner (Fig. 8). PI3K and PDK1, on the other hand, phosphorylated Akt2 at Ser474 and Thr309 respectively. This indicates that Akt2 may be activated by the cooperative mechanism of PI3K and PDK1 (Fig. 8). In this study, Akt2 was present at more than 200-fold greater levels than Akt1 in differentiated 3T3-L1 adipocytes. Then, the critical question is why insulin-induced Akt1/2 phosphorylation at Thr308/309 was not significantly inhibited by BX912 or knocking-down PDK1. A plausible explanation for this is that an anti-pT308(9) antibody might detect Akt1 phosphorylation at Thr308, even though expression levels of Akt1 were quite low. To answer this question, further experiments need to be carried out.

IKBKE and Pak1 have been shown to phosphorylate Akt at Ser473 in a PI3K-independent manner (Mao et al. 2008, Guo et al. 2011). PI3K, alternatively, activates mTORC2 (Razmara et al. 2013), and activated mTORC2 is capable of phosphorylating Akt at Ser473 (Bayascas \& Alessi 2005, Gao et al. 2005). In this study, PI3K phosphorylated Akt1/2 at Ser473/474 under cell-free conditions in the absence of mTORC2. This indicates

Published by Bioscientifica Ltd 
that phosphorylation of Akt1/2 at Ser473/474 could be achieved by PI3K alone, although the role of mTORC2 was not investigated. To our knowledge, this is the first study to detect direct phosphorylation of Akt1/2 at Ser473/474 due to PI3K.

The three Akt isoforms Akt1, Akt2, and Akt3 have critical and distinct functions in the regulation of metabolism, cell growth, and apoptosis. A growing body of evidence has argued that Akt2 is required for GLUT4 translocation. Insulin activates both Akt1 and Akt2 in 3T3-L1 and primary rat adipocytes (Bae et al. 2003,

A
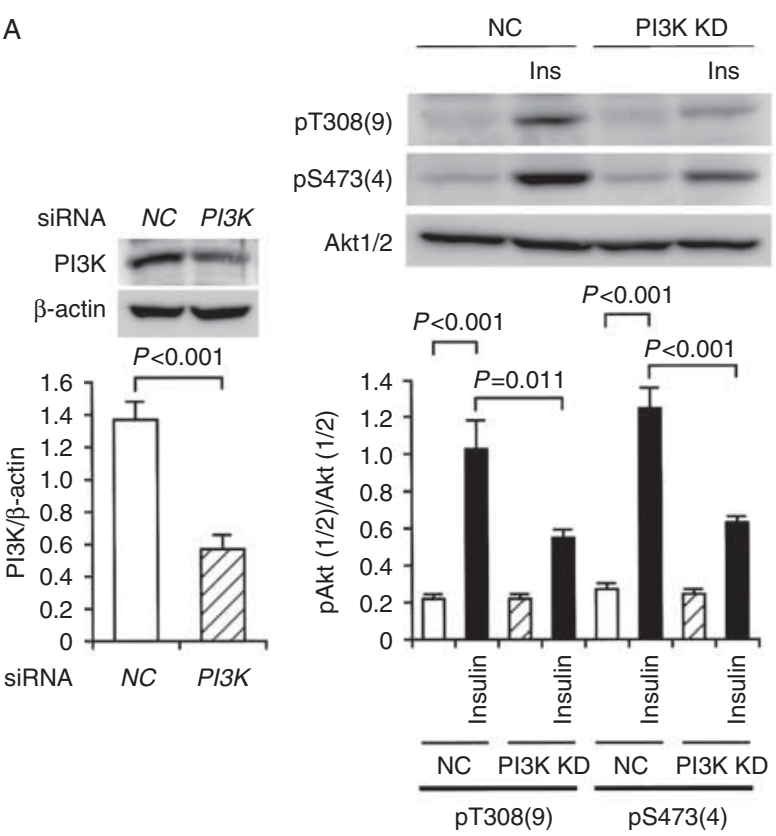

B
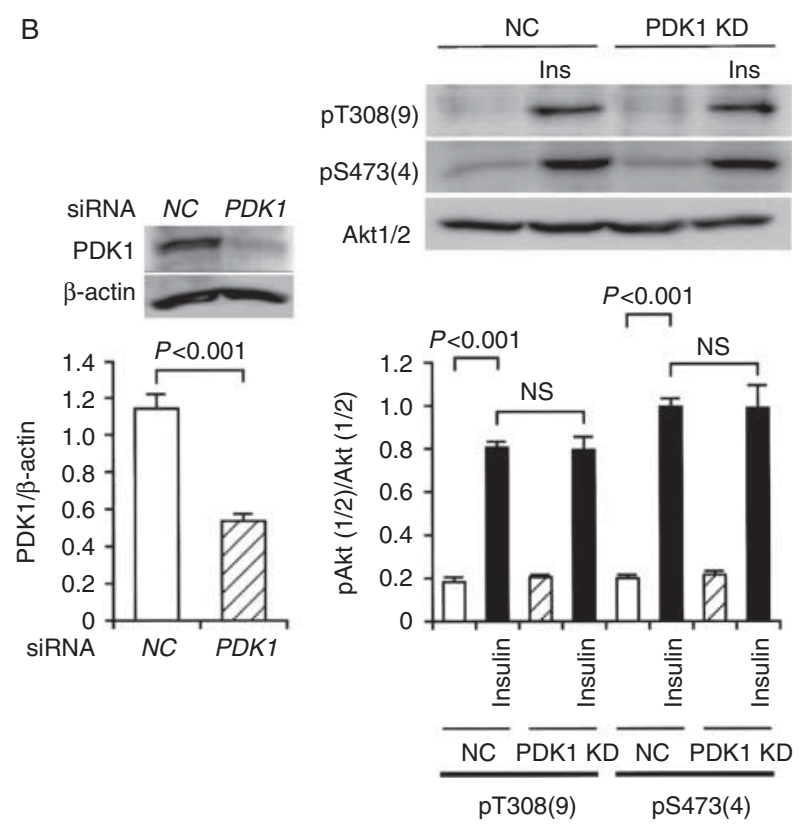

http://joe.endocrinology-journals.org DOI: 10.1530/JOE-13-0172

(c) 2014 The authors Printed in Great Britain
Gonzalez \& McGraw 2009). Isoform-specific activation, therefore, does not account for the selective requirement for Akt2 in the control of GLUT4 translocation to the plasma membrane in adipocytes. Akt2, but not Akt1, is associated with GLUT4 vesicles in biochemical fractionation of adipocytes (Calera et al. 1998, Hill et al. 1999), suggesting that differential localization allows Akt2 to control isoform-specific signaling. Moreover, insulin induces a preferential accumulation of Akt2, relative to Akt1, at the plasma membrane in adipocytes (Gonzalez \& McGraw 2009), indicating that regulation of GLUT4 translocation depends upon the amount of the isoform localization at the plasma membrane, but not the isoform species. In support of this notion, expression of an Akt1 mutant that accumulates at the plasma membrane is sufficient to induce GLUT4 translocation (Gonzalez \& McGraw 2009). Akt1, on the other hand, has also been shown to participate in the GLUT4 translocation to the plasma membrane in L6 myoblasts (Wang et al. 1999).

In this study, insulin-stimulated GLUT4 translocation to the cell surface in adipocytes was prevented by the Akt inhibitor MK2206 and knocking-down Akt1/2, the PI3K inhibitor wortmannin and knocking-down PI3K, or the PDK1 inhibitor BX912 and knocking-down PDK1. This indicates that insulin stimulates GLUT4 translocation to

\section{Figure 5}

Insulin-induced Akt1/2 phosphorylation at Thr308 and Ser473 was prevented by knocking-down PI3K, but not PDK1, in 3T3-L1-GLUT4myc adipocytes. (A) In the left panel, cells were transfected with the NC siRNA or the PI3K siRNA, and $48 \mathrm{~h}$ after transfection western blotting was carried out using antibodies against PI3K or $\beta$-actin. Signal intensities for PI3K were normalized to those for $\beta$-actin. In the graph, each column represents the mean ( \pm S.E.M.) normalized expression of PI3K ( $n=4$ independent experiments). $P$ value, unpaired $t$-test. In the right panel, cells transfected with the NC siRNA (NC) or the PI3K siRNA (PI3K KD) were left untreated or treated with insulin (Ins) (100 nM) for $10 \mathrm{~min}$, followed by western blotting using antibodies against pT308(9), pS473(4), and Akt1/2. Signal intensities for phosphorylated Akt1/2 (pAkt1/2) were normalized to those for Akt1/2. In the graph, each column represents the mean ( \pm s.E.M.) normalized intensity for $\mathrm{pAkt} 1 / 2$ at each site ( $n=4$ independent experiments). $P$ values, ANOVA followed by a Bonferonni correction. (B) In the left panel, cells were transfected with the NC siRNA or the PDK1 siRNA, and $48 \mathrm{~h}$ after transfection western blotting was carried out using antibodies against PDK1 or $\beta$-actin. Signal intensities for PDK1 were normalized to those for $\beta$-actin. In the graph, each column represents the mean ( \pm s.E.M.) normalized expression of PDK1 ( $n=4$ independent experiments). $P$ value, unpaired $t$-test. In the right panel, cells transfected with the NC siRNA (NC) or the PDK1 siRNA (PDK1 KD) were left untreated or treated with insulin (Ins) $(100 \mathrm{nM})$ for $10 \mathrm{~min}$, followed by western blotting using antibodies against pT308(9), pS473(4), and Akt1/2. Signal intensities for phosphorylated Akt1/2 (pAkt1/2) were normalized to those for Akt1/2. In the graph, each column represents the mean ( \pm s.E.M.) normalized intensity for pAkt1/2 at each site ( $n=4$ independent experiments). $P$ values, ANOVA followed by a Bonferonni correction. NS, not significant.

Published by Bioscientifica Ltd 

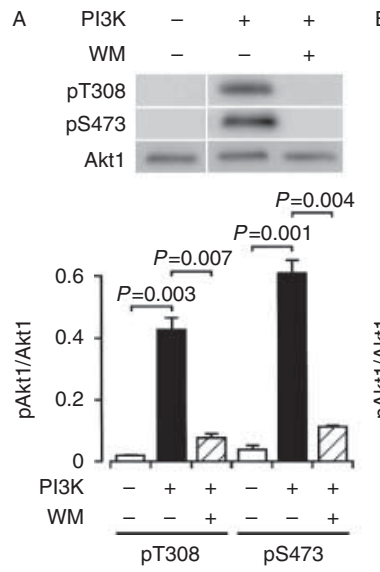

B
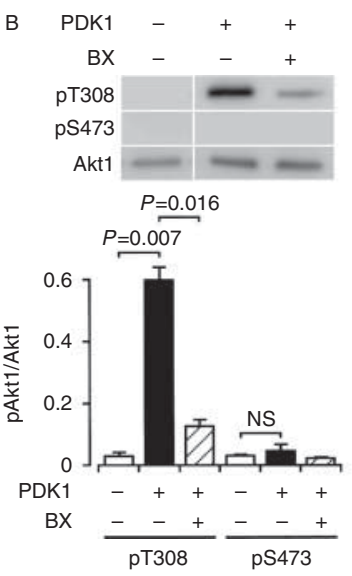

Figure 6

PI3K-dependent and PDK1-independent Akt1 phosphorylation and PI3K-/PDK1-dependent Akt2 phosphorylation under cell-free conditions. Akt1 or Akt2 was reacted with $(+)$ and without $(-)$ PI3K $(1 \mu \mathrm{g} / \mathrm{ml})(A$ and $C)$ or PDK1 $(1 \mu \mathrm{g} / \mathrm{ml})(B$ and $D)$ in the presence and absence of wortmannin (WM) (20 nM) or BX912 (BX) (100 nM), and western

the cell surface by activating Akt1/2 through a pathway along an IRS/PI3K/PDK1/Akt axis in adipocytes. Akt2 was abundantly expressed in adipocytes and Akt2 activation was achieved by cooperation of PI3K and PDK1 in the cellfree assay. This indicates that insulin dominantly activates Akt2 following activation of PI3K and the downstream effector PDK1 in adipocytes, responsible for GLUT4 translocation. Strangely, insulin-induced phosphorylation of Akt1/2 both at Thr308/309 and Ser473/474 in adipocytes was abrogated by wortmannin and knocking-down

A
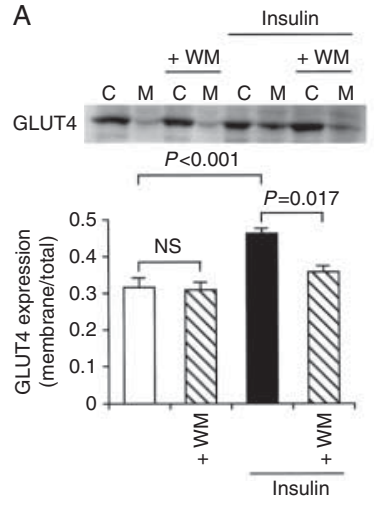

B

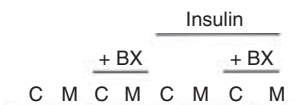
GLUT4

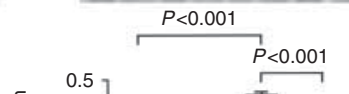

C
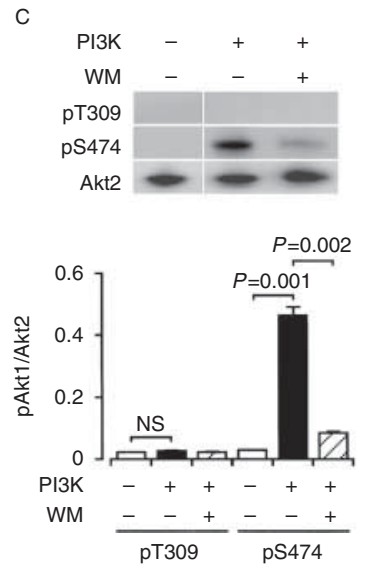

D

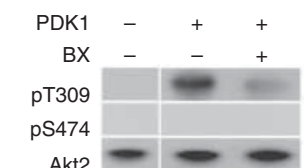

Akt2

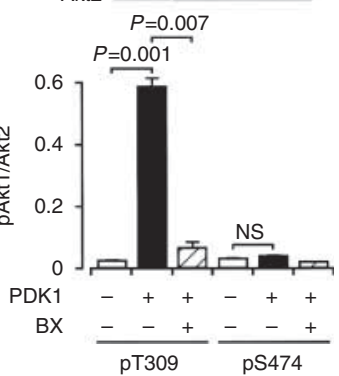

blotting was carried out using antibodies against pT308(9), pS473(4), and Akt1/2. Signal intensities for phosphorylated Akt1 (pAkt1) or Akt2 (pAkt2) were normalized to those for Akt1 or Akt2. In the graphs, each value represents the mean ( \pm S.E.M.) intensity for pAkt1 or pAkt2 at each site $(n=4)$. $P$ values, Dunnett's test. NS, not significant.

PI3K, but otherwise no significant inhibition of Akt1/2 phosphorylation at each site was obtained with BX912 and knocking-down PDK1. A plausible explanation for this is that PDK1 might stimulate GLUT4 translocation to the cell surface by phosphorylating or interacting with an unknown factor, as its downstream target, regardless of Akt1/2 phosphorylation at Thr308/309. To address this, we are currently carrying out further experiments.

In conclusion, the results presented here clearly demonstrate that insulin stimulates GLUT4 translocation

C
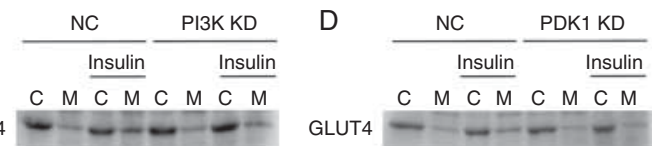

GLUT4
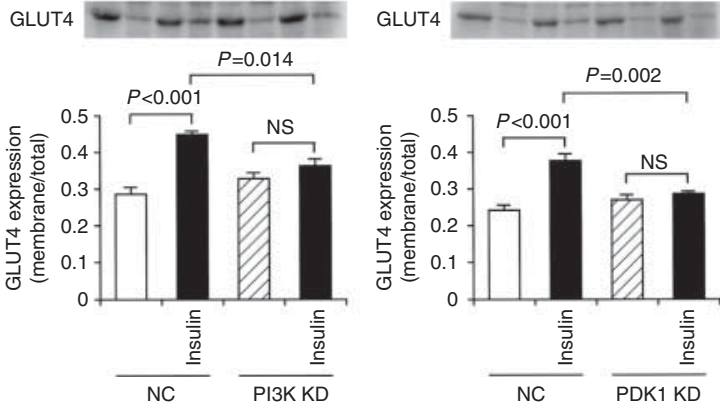

cells were lysed and separated into the cytosolic (C) and plasma membrane fractions (M), followed by western blotting using an antibody against c-myc. In the graphs, each column represents the mean ( \pm s.E.M.) ratio of signal intensity for c-myc in the plasma membrane fraction to that in the total cell ( $n=4$ independent experiments). $P$ values, ANOVA followed by a Bonferonni correction. NS, not significant. 


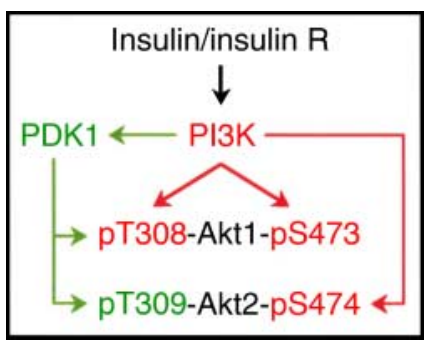

\section{Figure 8}

A schematic diagram of Akt activation pathway relevant to insulin signals. Insulin $\mathrm{R}$, insulin receptor.

to the plasma membrane in a PI3K-, PDK1-, and Akt1/2dependent manner in 3T3-L1-GLUT4myc adipocytes. The results also show that PI3K phosphorylates Akt1 both at Thr308 and Ser473 and Akt2 at Ser474 and that PDK1, a downstream effector of PI3K, phosphorylates Akt1 and Akt2 at Thr308 and Thr309 respectively. This implies that Akt1 is activated by PI3K alone and that Akt 2 activation is achieved by cooperation of PI3K and PDK1. The results of the present study, thus, may extend our understanding about Akt $1 / 2$ activation pathways linked to insulin signals.

\section{Declaration of interest}

The authors declare that there is no conflict of interest that could be perceived as prejudicing the impartiality of the research reported.

\section{Funding}

This research did not receive any specific grant from any funding agency in the public, commercial or not-for-profit sector.

\section{Author contribution statement}

A Tsuchiya and T Kanno performed all the experiments, analyzed the data, and prepared the figures. T Nishizaki designed the experiments and wrote the manuscript. All authors have approved the final manuscript.

\section{Acknowledgements}

We thank Prof. Ebina (Institute for Enzyme Research, The University of Tokushima, Tokushima, Japan) for providing us with 3T3-L1-GLUT4myc fibroblast cell line.

\section{References}

Bae SS, Cho H, Mu J \& Birnbaum MJ 2003 Isoform-specific regulation of insulin-dependent glucose uptake by Akt/protein kinase B. Journal of Biological Chemistry 278 49530-49536. (doi:10.1074/jbc.M306782200)

Bayascas JR \& Alessi DR 2005 Regulation of Akt/PKB Ser473 phosphorylation. Molecular Cell 18 143-145. (doi:10.1016/j.molcel.2005.03.020)
Beaulieu JM, Sotnikova TD, Marion S, Lefkowitz RJ, Gainetdinov RR \& Caron MG 2005 An Akt/ $\beta$-arrestin 2/PP2A signaling complex mediates dopaminergic neurotransmission and behavior. Cell 122 261-273. (doi:10.1016/j.cell.2005.05.012)

Bryant NJ, Govers R \& James DE 2002 Regulated transport of the glucose transporter GLUT4. Nature Reviews. Molecular Cell Biology 3 267-277. (doi:10.1038/nrm782)

Calera MR, Martinez C, Liu H, Jack AKE, Birnbaum MJ \& Pilch PF 1998 Insulin increases the association of Akt-2 with Glut4-containing vesicles. Journal of Biological Chemistry 273 7201-7204. (doi:10.1074/ jbc.273.13.7201)

Cheung M \& Testa JR 2013 Diverse mechanisms of AKT pathway activation in human malignancy. Current Cancer Drug Targets 13 234-244. (doi:10.2174/1568009611313030002)

Cong LN, Chen H, Li Y, Zhou L, McGibbon MA, Taylor SI \& Quon MJ 1997 Physiological role of Akt in insulin-stimulated translocation of GLUT4 in transfected rat adipose cells. Molecular Endocrinology 11 1881-1890. (doi:10.1210/me.11.13.1881)

Finlay DK 2012 Regulation of glucose metabolism in T cells: new insight into the role of phosphoinositide 3-kinases. Frontiers in Immunology 3 247. (doi:10.3389/fimmu.2012.00247)

Gao T, Furnari F \& Newton AC 2005 PHLPP: a phosphatase that directly dephosphorylates Akt, promotes apoptosis, and suppresses tumor growth. Molecular Cell 18 13-24. (doi:10.1016/j.molcel.2005.03.008)

Garofalo RS, Orena SJ, Rafidi K, Torchia AJ, Stock JL, Hildebrandt AL, Coskran T, Black SC, Brees DJ, Wicks JR et al. 2003 Severe diabetes, age-dependent loss of adipose tissue, and mild growth deficiency in mice lacking Akt2/PKBß. Journal of Clinical Investigation 112 197-208.

Gonzalez E \& McGraw TE 2009 Insulin-modulated Akt subcellular localization determines Akt isoform specific signaling. PNAS 106 7004-7009. (doi:10.1073/pnas.0901933106)

Guo JP, Coppola D \& Cheng JQ 2011 IKBKE protein activates Akt independent of phosphatidylinositol 3-kinase/PDK1/mTORC2 and the pleckstrin homology domain to sustain malignant transformation. Journal of Biological Chemistry 286 37389-37398. (doi:10.1074/jbc.M111.287433)

Hill MM, Clark SF, Tucker DF, Birnbaum MJ, James DE \& Macaulay SL 1999 A role for protein kinase B $\beta /$ Akt2 in insulin-stimulated GLUT4 translocation in adipocytes. Molecular and Cellular Biology 19 7771-7781.

Ishiki M \& Klip A 2005 Minireview: recent developments in the regulation of glucose transporter- 4 traffic: new signals, locations, and partners. Endocrinology 146 5071-5078. (doi:10.1210/en.2005-0850)

Ishikura S, Koshkina A \& Klip A 2008 Small G proteins in insulin action: Rab and Rho families at the crossroads of signal transduction and GLUT4 vesicle traffic. Acta Physiologica 192 61-74. (doi:10.1111/j.17481716.2007.01778.x)

Lai KM, Gonzalez M, Poueymirou WT, Kline WO, Na E, Zlotchenko E, Stitt TN, Economides AN, Yancopoulos GD \& Glass DJ 2004 Conditional activation of Akt in adult skeletal muscle induces rapid hypertrophy. Molecular and Cellular Biology 24 9295-9304. (doi:10.1128/MCB.24.21.9295-9304.2004)

Maarbjerg SJ, Sylow L \& Richter EA 2011 Current understanding of increased insulin sensitivity after exercise-emerging candidates. Acta Physiologica 202 323-335. (doi:10.1111/j.1748-1716.2011.02267.x)

Mao K, Kobayashi S, Jaffer ZM, Huang Y, Volden P, Chernoff J \& Liang Q 2008 Regulation of Akt/PKB activity by P21-activated kinase in cardiomyocytes. Journal of Molecular and Cellular Cardiology 44 429-434. (doi:10.1016/j.yjmcc.2007.10.016)

Ramírez-Zacarías JL, Castro-Muñozledo F \& Kuri-Harcuch W 1992 Quantitation of adipose conversion and triglycerides by staining intracytoplasmic lipids with Oil red O. Histochemistry 97 493-497. (doi:10.1007/BF00316069)

Razmara M, Heldin C-H \& Lennartsson J 2013 Platelet-derived growth factor-induced Akt phosphorylation requires mTOR/Rictor and phospholipase C- $\gamma 1$, whereas S6 phosphorylation depends on mTOR/Raptor and phospholipase D. Cell Communication and Signaling 11 3. (doi:10.1186/1478-811X-11-3) 
Rudich A \& Klip A 2003 Push/pull mechanisms of GLUT4 traffic in muscle cells. Acta Physiologica Scandinavica 178 297-308. (doi:10.1046/j.1365201X.2003.01163.x)

Song G, Ouyang G \& Bao S 2005 The activation of Akt/PKB signaling pathway and cell survival. Journal of Cellular and Molecular Medicine 9 59-71. (doi:10.1111/j.1582-4934.2005.tb00337.x)

Tontonoz P, Hu E \& Spiegelman BM 1994 Stimulation of adipogenesis in fibroblasts by PPAR $\gamma 2$, a lipid-activated transcription factor. Cell 79 1147-1156. (doi:10.1016/0092-8674(94)90006-X)

Vanhaesebroeck B \& Alessi DR 2000 The PI3K-PDK1 connection: more than just a road to PKB. Biochemical Journal 346 561-576. (doi:10.1042/ 0264-6021:3460561)
Wang Q, Somwar R, Bilan PJ, Liu Z, Jin J, Woodgett JR \& Klip A 1999 Protein kinase B/Akt participates in GLUT4 translocation by insulin in L6 myoblasts. Molecular and Cellular Biology 19 $4008-4018$.

Watson RT, Kanzaki M \& Pessin JE 2004 Regulated membrane trafficking of the insulin-responsive glucose transporter 4 in adipocytes. Endocrine Reviews 25 177-204. (doi:10.1210/er.2003-0011)

Yang J, Cron P, Thompson V, Good VM, Hess D, Hemmings BA \& Barford D 2002 Molecular mechanism for the regulation of protein kinase B/Akt by hydrophobic motif phosphorylation. Molecular Cell 9 1227-1240. (doi:10.1016/S10972765(02)00550-6)

Received in final form 11 October 2013

Accepted 29 October 2013

Accepted Preprint published online 29 October 2013
Published by Bioscientifica Ltd 